B20

\title{
Formation Evaluation and Reservoir Monitoring behind Dual Casings - A First Libya's Case Study
}

R.G. Gauchet* (CPTL), C.R. Robert (CPTL), M.A. Attayeb (CPTL) \& L.W. Wihardjo (Schlumberger)

\section{SUMMARY}

The success of Formation Evaluation and Reservoir Monitoring behind dual casings can be achieved with modern tools, provided proper planning \& logging preparation.

The casing ex-centralization and well deviation are key factors that provide tool orientation and make it possible to record a stronger formation signal.

The quantitative water saturation comparison (between open and cased hole evaluation) has indicated significant remaining oil left at the Dahra formation. The current oil water contact has been positively identified. 


\section{Introduction}

In order to ensure a proper reservoir data acquisition with the objective to set up a field development in the Sirte basin, CPTL was faced with the challenge of acquiring crucial data behind dual casings in a vertical well drilled in the 60's, and put on long production test in the 90's. The main objectives were first to have a consistent petrophysical description (especially porosity) and second to define the current fluid distribution within the reservoir after about 7 years of production (remaining oil and water oil contact evolution). Pulse Neutron Capture (PNC) and Pulse Neutron Spectroscopy (PNS) were chosen to tackle this challenge.

To perform a successful Cased Hole logging, the following parameters have to be considered during the planning stage: formation property - lithology and porosity -, formation water salinity, hole size and well completion configuration (casing size \& weight). In the Mabruk concession (C-17), the well was completed with dual 7 and $95 / 8$ inches casings. The well intersected two different formations that needed to be surveyed :

- Mabruk formation (upper section, secondary objective) : $40 \mathrm{gr} / \mathrm{l} \mathrm{NaCl}$ equiv. formation water salinity, low to medium porosity (average $<15 \mathrm{PU}$ )

- Dahra formation (lower section, primary objective) : $65 \mathrm{gr} / 1 \mathrm{NaCl}$ equiv. formation water salinity, medium to high porosity ( average $>18 \mathrm{PU}$ )

The only Open Hole log data available are Gamma Ray (GR), Spontaneous Potential (SP) and Induction $\log$, which is not sufficient to carry out a complete Archie Based Saturation Evaluation, mainly due to the absence of porosity data.

\section{Dual Casings Cased Hole Logging}

Knowing the challenges, and given the absence of porosity data, a set of cased hole measurements was defined, and then acquired :

- CBL-VDL-USI to provide a complete estimation of cement bond quality. CBL-VDL will give the standard bonding quality (which is omni direction) whereas USI will provide the detailed picture of cement distribution between the two casings.

- APS to provide porosity log behind casing. APS is less affected by borehole (Temp., Salinity, Standoff) than conventional CNL.

- RST-D in both Sigma (Pulse Neutron Capture) \& Carbon Oxygen (Pulse Neutron Spectroscopy) modes to evaluate remaining oil and oil water contact. RST Sigma is salinity dependent, and therefore is better suited to evaluate remaining oil when formation water is saline enough ( $>50,000$ ppm salinity), whereas Carbon Oxygen is salinity independent. Both Sigma and Carbon Oxygen have a shallow depth of investigation (8-12 inches for Sigma mode and 6-8 inches for Carbon Oxygen mode).

\section{Observations}

The cased hole logging result can be summarized as follows:

- $C B L-V D L-U S I$

- Casing ex-centralization is indicated by the USI "galaxy" pattern. It confirms that this well might be slightly deviated.

- Possible channeling between casings can be seen from the USI cement distribution map. 
- Zonal isolation can be observed by combining CBL-VDL and USI. VDL Formation arrival confirms good bonding between outer casing ( $9 \frac{5}{8}$ inch) to formation.

- $A P S$

- Due to the slight well deviation, and the fact that casing is not centralized, the tool was ex-centralized, lying down on the bottom part of the well. This lead to a stronger signal from the formation in the short axis direction.

- Good porosity measurements were provided and match with the core porosity measurement.

- Good comparison between the APS porosity and the RST porosity measurements is observed and increase the confidence in the measurements

- RST-D Sigma \& Carbon Oxygen

- The casing ex-centralization observed on the USI was beneficial for the RST measurement. The tool laid down in the short axis, which allowed for more formation signal to be captured.

- Sigma method gave a good contrast between water (40 g/l \& $65 \mathrm{~g} / 1)$ against the oil signature. Water Saturation $(\mathrm{Sw})$ can be computed by using Sigma Saturation Equation.

- Carbon Oxygen method gave enough Carbon Oxygen (FCOR, FWCO) ratio contrast between oil and water. Oil Saturation (So) can be obtained by using Schlumberger RST C/O Processing Chained in GeoFrame 4.2.

- Both measurements (Sigma \& Carbon Oxygen) have a good agreement on remaining oil and water contact.

\section{Conclusions}

- The success of this operation shows that the Formation Evaluation and Reservoir Monitoring behind dual casings can be achieved with modern tools, provided proper planning \& logging preparation

- The casing ex-centralization and well deviation are key factors that provide tool orientation and make it possible to record a stronger formation signal.

The quantitative water saturation comparison (between open and cased hole evaluation) has indicated significant remaining oil left at the Dahra formation. The current oil water contact has been positively identified. 